\title{
Phase Space Volumes of Non-Neutral Electron Plasmas during Vortex Merging Processes
}

\author{
Chikato KAGA, Yukihiro SOGA ${ }^{1)}$, Kiyokazu ITO, Hiroyuki HIGAKI and Hiromi OKAMOTO \\ Graduate School of Advanced Sciences of Matter, Hiroshima University, \\ 1-3-1 Kagamiyama, Higashi-Hiroshima 739-8530, Japan \\ 1) Faculty of Mathematics and Physics, Institute of Science and Engineering, Kanazawa University, \\ Kanazawa 920-1192, Japan
}

(Received 20 October 2014 / Accepted 17 December 2014)

\begin{abstract}
Non-neutral electron plasmas in a uniform magnetic field are investigated experimentally as a two dimensional fluid. It is confirmed that the phase space volume increases during a vortex merging process, which is consistent with the presence of viscosity. It is also suggested that the following vortex holes movement is associated with a dissipative process.
\end{abstract}

(C) 2015 The Japan Society of Plasma Science and Nuclear Fusion Research

Keywords: non-neutral plasma, 2D fluid, vortex merging, vortex holes, phase space

DOI: $10.1585 /$ pfr. 10.1201004

Non-neutral electron plasmas confined in a uniform magnetic field have been studied as 2 dimensional (2D) fluids [1-6]. Assuming the correspondence between the 2D drift-Poisson equation and the 2D Euler equation [1], non-neutral electron plasmas are treated as ideal 2D (inviscid) Euler fluids. It means for non-neutral electron plasmas that the cyclotron motion of electrons in the magnetic field is neglected by the guiding center approximation, that the collisions between electrons are neglected, and that the effects of confining potentials are negligible. In fact, when the system is approximated as two point vortices [3,4], it was confirmed experimentally that the system followed the Hamiltonian formulation by Onsager [7]. Thus, a trajectory of vortex motion is restricted to a constant Hamiltonian. Also, various point vortex crystals were studied with and without background vorticity [6,8-11]. Merging processes of vortices were also studied with special emphasis on the geometrical structure [2, 10, 11] and viscosity [12]. In Ref. [12], a global viscosity is estimated with enstrophy and palinstrophy. The presence of viscosity during the vortex merging process suggests that 2D Euler picture is not applicable. Here, a similar vortex merging process is investigated experimentally from different view points, i.e., an intensity (vorticity) histogram and a phase space volume.

The experimental apparatus is composed of an electron gun, solenoidal coils, 45 ring electrodes and phosphor screen with CCD camera. Electrons are confined inside a set of ring electrodes with the inner diameter of $70 \mathrm{~mm}$ in a uniform axial magnetic field $B_{z} \sim 250 \mathrm{G}$. Potential well formed by negative voltages applied to both ends of electrodes provides axial confinement and the magnetic field provides radial confinement. An initial state of hollow den-

author'se-mail:d146073@hiroshima-u.ac.jp
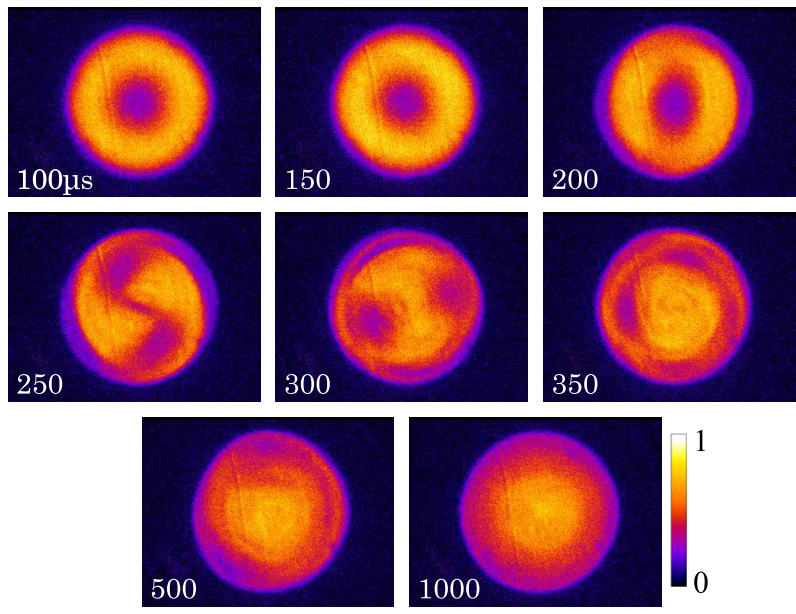

Fig. 1 Images of the intensity ( $\propto$ vorticity) distribution for various confinement time $t$. The density is normalized by the peak intensity of each image for the contrast.

sity distribution is established by reducing potential barrier briefly at the injection side. The trapped electron vortex has length $L_{\mathrm{p}} \sim 500 \mathrm{~mm}$, with the inner and outer radius of $\sim 5 \mathrm{~mm}$ and $\sim 15 \mathrm{~mm}$, respectively. Typical peak density of electrons is $n \sim 10^{12} \mathrm{~m}^{-3}$.

Figure 1 shows the single shot phosphor screen images when electrons are dumped after a certain confinement time $t$. The color scale is normalized to each peak intensity. The pictures show the time evolution from $t=100$ to $350 \mu \mathrm{s}(50 \mu \mathrm{s}$ intervals $)$ and $t=500,1000 \mu \mathrm{s}$. The hollow distribution remains until $t=150 \mu \mathrm{s}$ and deforms due to the onset of $m=2$ diocotron instability at $t=200 \mu \mathrm{s}$. Then, the distribution becomes two clumps at $t=250 \mu \mathrm{s}$, which approach each other and merge at $t=300 \mu \mathrm{s}$, and 

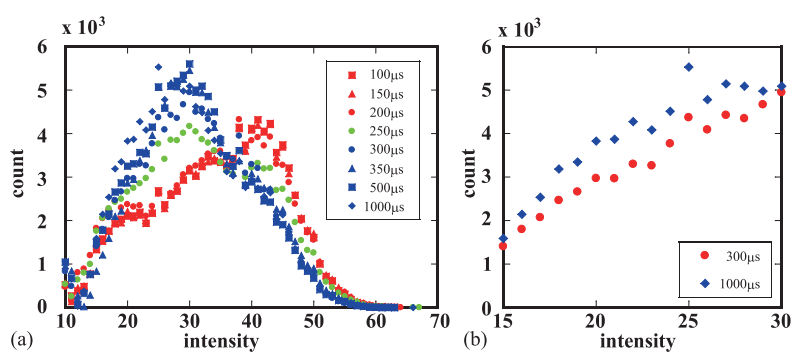

Fig. 2 (a) Intensity ( $\propto$ vorticity) histograms at various $t$. Red symbols show the histograms before merging, and blue symbols show the histograms after merging. (b) the histogram magnified at intensity between 15 and 30 for $t=300$ and $1000 \mu \mathrm{s}$.

concentrates near the center at $t \sim 350 \mu \mathrm{s}$. Afterwards, vortex holes move radially outward [13]. In the theory of 2D point vortex system [7], the point vortex Hamiltonian $H$ results in the equations of motion for a point vortex with a local vorticity $\Gamma_{k} . \Gamma_{k} \frac{\mathrm{d} x_{k}}{\mathrm{~d} t}=\frac{\partial H}{\partial y}$ and $\Gamma_{k} \frac{\mathrm{d} y_{k}}{\mathrm{~d} t}=-\frac{\partial H}{\partial x}$. These equations correspond to Hamilton's canonical equations. Therefore, canonical variables in the $2 \mathrm{D}$ vortex system becomes space coordinate $(x, y)$. And the phase space volume occupied by point vorticies corresponds to the area in $x-y$ plain occupied by vorticies. In 2D Euler fluid, the area becomes constant under a fluid motion (Liouville theorem).

In Fig. 2 (a), the time evolution of intensity (vorticity) histograms are plotted. The intensity less than 10 is neglected because there are systematically negative values when background noises are subtracted. If there is no viscosity, the histogram should keep its profile. It is clearly seen that the profile of the intensity histogram changes before (red) and after (blue) the vortex merging process $(t=200 \sim 300 \mu \mathrm{s})$. High intensity components around 40 decrease and low intensity components around 30 increase. Also shown in Fig. 2 (b) is the expanded histogram at intensity between 15 and 30 for $t=300$ and $1000 \mu$ s. After the vortex merging, intensity in this region increases associated with the outward movement of vortex holes.

During the course of vortex evolution, three integrals calculated from observed intensity distributions are important. The total electron number $N_{\text {tot }}=\int \mathrm{d}^{2} \boldsymbol{r} n(\boldsymbol{r})$, the electrostatic energy $E_{\text {tot }}=1 / 2 \int \mathrm{d}^{2} \boldsymbol{r}(-e) \phi(\boldsymbol{r}) n(\boldsymbol{r})$, and the angular momentum $P_{\theta}=\int \mathrm{d}^{2} \boldsymbol{r}(-e B / 2) r^{2} n(\boldsymbol{r}) . N_{\text {tot }}, E_{\text {tot }}$ and $P_{\theta}$ are equivalent to total circulation, fluid kinetic energy and angular impulse which are constants of 2D Euler fluid. These quantities normalized to unity are plotted in Fig. 3 (a).

Solid circles in Fig. 3 (a) shows the total number of the pixel $N_{\mathrm{p}}$ (which corresponds to area in the phase space) where vorticity exists. It is clear that the total number of pixels increases during a merging process (between $200 \mu \mathrm{s}$ and $300 \mu \mathrm{s}$ ). This results support that $2 \mathrm{D}$ Euler fluid model is inapplicable for the current vortex merging processes.
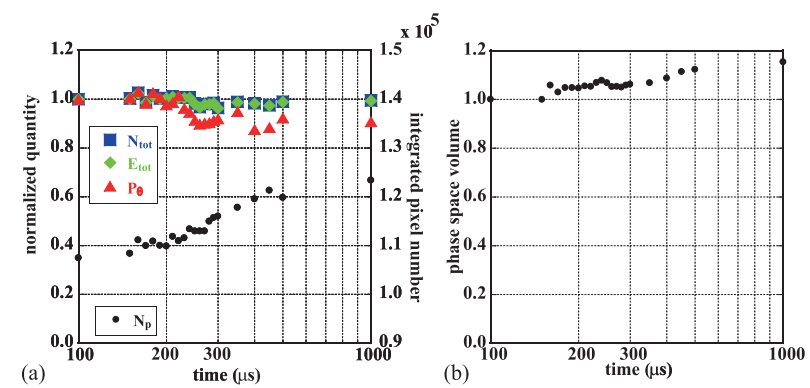

Fig. 3 (a) The time evolution of $N_{\text {tot }}$ (blue squares), $E_{\text {tot }}$ (green diamonds), and $P_{\theta}$ (red triangles). The solid circles are the integration of vorticity profiles, which corresponds to $2 \mathrm{D}$ phase space area. (b) The time evolution of $4 \mathrm{D}$ phase space volume.

Furthermore, it is inferred that the outward motion of vortex holes is also associated with a dissipative process in this case as seen by Fig. 2(b). Although the influence of coarse graining may be included as a part of reasons for increment of $N_{\mathrm{p}}$, it is difficult to separate dissipation from coarse graining by experiments.

Here, the 4D phase space volume is also estimated by $V_{\mathrm{ps}}=\sum_{i} \sum_{j} \mathrm{~d} x_{i} \mathrm{~d} P_{x_{i}} \mathrm{~d} y_{j} \mathrm{~d} P_{y_{j}}$ with the canonical momentum $P_{x_{i}}$ and $P_{y_{i}}$. This is the sum of differences of the position and canonical momentum between the pixels in which electron exists. Shown in Fig. 3 (b) is the 4D phase space volume as a function of time normalized with the value of $100 \mu \mathrm{s}$. Although it seems the volume is conserved during the vortex merging at first glance, a careful estimation is necessary since the intensities less than 10 are ignored. Comparing the volume at $t \leq 200 \mu \mathrm{s}$ with that of $1000 \mu \mathrm{s}$, it is suggested that dissipation (and/or coarse graining) increases the 4D phase space volume for electrons.

In summary, the phase space volumes of nonneutral electron plasmas during vortex merging processes were evaluated experimentally for the first time. It was confirmed that both the $2 \mathrm{D}$ phase space volume as a fluid and $4 \mathrm{D}$ phase space as a plasma increase during the vortex merging processes, which is consistent with the presence of viscosity. Especially the outward movement of vortex holes following the vortex merging is also associated with a dissipation and/or coarse graining. Simulation studies are planned for the detailed investigations of these processes.

This work is partly supported by the JSPS KAKENHI Grant No.24340142.

[1] C.F. Driscoll and K.S. Fine, Phys. Fluids B2, 1359 (1990).

[2] K.S. Fine, C.F. Driscoll, J.H. Malmberg and T.B. Mitchell, Phys. Rev. Lett. 67, 588 (1991).

[3] T.B. Mitchell, C.F. Driscoll and K.S. Fine, Phys. Rev. Lett. 71, 1371 (1993).

[4] T.B. Mitchell and C.F. Driscoll, Phys. Fluids 8, 1828 (1996).

[5] D.L. Eggleston, Phys. Plasmas 1, 3850 (1994).

[6] K.S. Fine, A.C. Cass, W.G. Flynn and C.F. Driscoll, Phys. 
Rev. Lett. 75, 3277 (1995).

[7] L. Onsager, Nuovo Cimento, Supplemento 6, 279 (1949).

[8] Y. Kiwamoto, K. Ito, A. Sanpei and A. Mohri, Phys. Rev. Lett. 85, 3173 (2000).

[9] K. Ito, Y. Kiwamoto and A. Sanpei, Jpn. J. Appl. Phys. Part 1 40, 2558 (2001).

[10] A. Sanpei, Y. Kiwamoto, K. Ito and Y. Soga, Phys. Rev. E
68, 016404 (2003).

[11] Y. Kiwamoto, N. Hashizume, Y. Soga, J. Aoki and Y. Kawai, Phys. Rev. Lett. 99, 115002 (2007).

[12] Y. Kawai, Y. Kiwamoto, Y. Soga and J. Aoki, Phys. Rev. E 75, 066404 (2007).

[13] X.-P. Huang, K.S. Fine and C.F. Driscoll, Phys. Rev. Lett. 74, 4424 (1995). 